\title{
Influence of Polymer Morphology on the Capacity of Molecularly Imprinted Resins to Release or to Retain their Template
}

\author{
By Richard VENDAMME, ${ }^{1, *}$ Walter EEVERS, ${ }^{1}$ Masayuki KANETo, ${ }^{1}$ and Yoshihiro MINAMIZAKI ${ }^{2}$
}

\begin{abstract}
This paper deals with the specific ability of molecularly imprinted polymers (MIPs) to release and/or to retain their template. MIPs for caffeine with various MIP:porogen volume ratios were prepared by a non-covalent route. Formation of a prepolymerization complex between the template and the functional monomer was confirmed by nuclear magnetic resonance and computer simulation. The MIP monoliths were successively grinded, sieved, and dried under vacuum. The morphology of the imprinted particles was investigated by scanning electron microscopy, swelling experiments and nitrogen adsorption. Typical release patterns of as-synthesised MIP particles in solvents can be decomposed in three fractions: initial release $\left(\phi_{0}\right)$, slow release over several hours $\left(\Delta \phi^{\text {Release }}\right)$, permanent retention/encapsulation within the MIP $\left(\Delta \phi^{\text {Reservoir }}\right)$. The effect of MIP:porogen volume ratio (related to the MIP porosity) on the releasing/retaining profiles of the materials has been investigated. Finally, the results were critically compared with the available literature and the applicability of MIP reservoirs is briefly discussed.

KEY WORDS: Molecular Imprinting / Polymer Morphology / Controlled Release / Polymer Reservoir / Self-Assembly /
\end{abstract}

Molecular imprinting is an emerging technology, which allows the molecular design of polymeric materials containing highly specific receptor sites with an affinity for target compounds. ${ }^{1-3}$ Formation of a self-assembled pre-polymerization complex between a template molecule and functional monomers, followed by polymerization with a cross-linker in a porogenic solvent and the subsequent extraction of the template, produces a molecularly imprinted polymer (MIP) possessing specifically sized and shaped cavities capable of molecular recognition. ${ }^{4,5}$ MIPs are generally highly crosslinked thermosets, and therefore porosity is a necessary feature of their morphology to allow permeability and transport of templates molecules to the bulk polymer phase.

To date, a number of promising applications have been proposed for MIPs. For instance, imprinted polymers are used in chromatography as the stationary phase for separation and isolation of racemates. ${ }^{6,7} \mathrm{~A}$ related area is solid-phase extraction, where imprinted polymers are employed as a selective sorbent to concentrate the molecule of interest. ${ }^{8}$ MIPs are also used in immunoassays-type analyses as synthetic antibody $^{9}$ and in catalysis as enzyme mimics. ${ }^{10,11}$ In the emerging field of biosensors, MIPs demonstrate a potential as the recognition element, although their performance and selectivity in aqueous systems is still a limiting factor. ${ }^{12,13}$

The application of desorption properties of MIPs is a relatively new area. ${ }^{14,15}$ Chromatographic data have shown that the MIP-template dissociation kinetics can be controlled by varying the local environment (MIP composition, solvent...). Therefore, it seems feasible that MIPs could form highly specific rate limiting reservoirs releasing active compounds at well-defined rates. Early studies delivered promising results and showed that these affinity materials could be used for the controlled delivery of drugs ${ }^{16,17}$ or pesticides. ${ }^{18}$ Such MIP containers may have some applications in medical patches, therapeutic wound healing, and various types of industrial tapes and coatings possessing a modulated retaining function for active substances. Up to now, several research groups have investigated the release behavior of imprinted polymer particles that have been previously extensively washed and reloaded with a small amount of template.

Although binding and selectivity by MIPs in chromatographic or batch rebinding mode may not depend strongly on macroporosity, applications in controlled release and drug delivery may rely on mass-transfer kinetics related to polymer porosity. ${ }^{19,20}$ To our knowledge, the influence of polymer morphology and surface area on the release behavior of MIPs has not been reported yet. This work is part of our efforts to investigate the feasibility of functional MIP systems. In view of the potential use of MIPs as reservoirs of active substance and in controlled delivery vehicles, we have investigated the release behavior of as-synthesized MIP particles (direct release). In order to maximize the potential for additional future applications, the non-covalent approach to molecular imprinting has been employed. As a model system, MIP for caffeine was synthesized following the procedures reported in the literature. The release profiles of the MIPs have been investigated as a function of time and MIP composition (porosity), and the effective loading capacity of the materials has been evaluated from the release experiments.

\footnotetext{
${ }^{1}$ Nitto Denko Corporation's European Research Committee (ERC), Nitto Europe NV, 22 Eikelaarstraat, 3600 Genk, Belgium

${ }^{2}$ Production Engineering Development Centre, Nitto Denko Corporation 18, Azahirayama, Nakahara-cho, Toyohashi 441-3194, Japan

*To whom correspondence should be addressed (Tel: +32-(0)89-36 04 95, Fax: +32-(0)89-36 22 42, E-mail: richard_vendamme@ nittoeur.com).
} 
Table I. Composition of the pre-polymerisation MIP formulations ${ }^{a}$

\begin{tabular}{|c|c|c|}
\hline $\begin{array}{l}\text { Sample } \\
\text { Code }\end{array}$ & $\begin{array}{l}\text { Caffeine:MAA: } \\
\text { EGDMA (mol \%) }\end{array}$ & $\begin{array}{c}\text { Porogen (vol \% } \\
\text { MIP:porogen) }\end{array}$ \\
\hline NIP1 & $0: 4: 20$ & $\operatorname{ACN}(3: 4)$ \\
\hline NIP2 & $0: 4: 20$ & $\mathrm{ACN}(3: 2)$ \\
\hline MIP1 & $1: 4: 20$ & $\operatorname{ACN}(3: 4)$ \\
\hline MIP2 & $1: 4: 20$ & $\mathrm{ACN}(3: 2)$ \\
\hline MIP3 & $1: 4: 20$ & $\mathrm{CHCL}_{3}(3: 4)$ \\
\hline MIP4 & $1: 4: 20$ & $\mathrm{CHCL}_{3}(3: 2)$ \\
\hline MIP5 & $1: 4: 20$ & $\mathrm{CHCL}_{3}(3: 1)$ \\
\hline MIP6 & $1: 4: 10$ & ACN (3:4) \\
\hline MIP7 & $1: 4: 30$ & $\operatorname{ACN}(3: 4)$ \\
\hline
\end{tabular}

a The amount of initiator (AIBN) was $1 \%$ relative to the number of moles of polymerizable groups.

\section{EXPERIMENTAL}

\section{Materials}

Caffeine, chloroform (HPLC grade), and methanol (HPLC grade) were purchased from Sigma. Acetonitrile (ACN) was of HPLC grade and obtained from Riedel-de Haën. The functional monomer methacrylic acid (MAA) and the cross-linker ethylene glycol dimethacrylate (EGDMA) were both purchased from Aldrich. The initiator azobisizobutyronitrile (AIBN) was obtained from Arkema. All the chemicals were used as received without further purification.

\section{Polymer Preparation}

The template, functional monomer, crosslinker and initiator were dissolved into the porogenic solvent in a $200 \mathrm{~mL}$ glass flask with screw-cap (see Table I for the details of the formulations). The reaction mixtures were sonicated for $5 \mathrm{~min}$. $20 \mathrm{~mL}$ of the mixture was transferred into Pyrex Reusable Glass Tubes $(20 \times 150 \mathrm{~mm})$ with PTFE-Faced Rubber-Lined Caps. The tubes were then sealed under nitrogen and the polymerization was conducted for $24 \mathrm{~h}$ on a custommade photo-polymerisation device equipped with a temperature control-unit. Bulk polymers were extracted from the reaction flask and ground manually with pestle and mortar. The MIP particles were then sieved using a Retsch vibratory analytical sieve shaker (AS 200) and dried under vacuum at room temperature.

In order to check the gravimetric yield of polymerization, $20 \mathrm{~mL}$ of a reactive MIP formulation was polymerized in a Pyrex tube as described above. The bulk polymer was roughly fragmented and transferred into $100 \mathrm{~mL}$ of fresh acetonitrile. After an extraction time of 2 weeks, the polymer fragments were removed with a syringe equipped with a PTFE filter, and the quantitative analysis of MAA and EGDMA in the liquid phase was performed by HPLC (UV-vis detection). As a reference, $20 \mathrm{~mL}$ of the un-polymerized MIP formulation was directly added to $100 \mathrm{~mL}$ of acetonitrile and similarly analyzed by HPLC.

\section{Apparent Density and Apparent Swelling of the MIP Particles}

The apparent density of the dried MIP particles was measured by introducing a given volume of MIP powder (from $0,5 \mathrm{~mL}$ to $1 \mathrm{~mL}$ ) in a volume-calibrated NMR tube and by measuring the corresponding polymer weight. The particles were packed down in the tube by centrifugation (Hettich Universal 320; $2000 \mathrm{rpm}, 30 \mathrm{~min}$ ) prior to measuring the exact volume. For the apparent swelling studies, the particles were immersed in an excess of solvent and allowed to swell freely for $24 \mathrm{~h}$. The volumes of the particles in the dry and swollen states were both measured after $30 \mathrm{~min}$ of centrifugation (2000 rpm).

\section{Characterization}

${ }^{1} \mathrm{H}$ NMR was performed to study the strength of the interactions between caffeine with MAA in acetonitrile and chloroform. Caffeine and MAA were dissolved in the deuterated solvents to get their respective ${ }^{1} \mathrm{H}$ NMR spectra. Then caffeine and MAA were mixed according to the molar ratio of the pre-polymerization mixture. The spectrum of the mixture was determined after shaking for few hours to ensure a complete interaction of caffeine with MAA. All NMR spectra were acquired on a $400 \mathrm{MHz}$ Varian Inova spectrometer.

Polymers were imaged using a Quanta 200-FEG/EDX raster electron microscope. Specimens were sputtered with a thin conducting layer to reduce load up of the sample. Secondary electrons (SE) were used for obtaining pictures of the surfaces morphology under study. Nitrogen adsorption measurements were performed using a Quadrasorb SI (Quantachrome) automated gas sorption system. Prior to the measurements, the samples were outgassed under vacuum at $170^{\circ} \mathrm{C}$ during $16 \mathrm{~h}$ on an Autosorb Degasser (Quantachrome). After outgassing, the sample cell is filled with helium to bring it to atmosphere and detached from the outgasser. The sample was then attached to the quadrasorb equipment and the nitrogen sorption measurement is performed at $77 \mathrm{~K}$. The Barret-JoynerHalenda (BJH) method applied on the adsorption branch of the isotherm was used to determine the pore size distribution and the Brunauer-Emmet-Teller (BET) method was applied to calculate the specific surface area. The total pore volume was determined at a relative pressure of 0,98 .

\section{MIP Release Experiment}

At first, standard calibration equations for caffeine in various release solvents were established by measuring the intensity of the absorbance peak at $273 \mathrm{~nm}$ for concentrations ranging from $10 \mathrm{mg} / \mathrm{L}$ to $50 \mathrm{mg} / \mathrm{L}$. The release profile of the sample MIP1 in ACN was performed as follows. A dispersion of as-synthesised, dried MIP particles in the release solvent was prepared in a glass bottle with screw-cap. The respective amounts of MIP powder and solvent were calculated in order to obtain a total concentration of template in the dispersion of $50 \mathrm{mg} / \mathrm{L}$ (typically $10 \mathrm{mg}$ of template in $200 \mathrm{~mL}$ of solvent). The weight of dried MIP particles corresponding to $10 \mathrm{mg}$ of template was determined from the template weight fraction in the MIP precursor formulation. In fact, assuming a complete mass conservation of the polymer precursors after polymerization, the template weight fraction should be constant both in 
the initial MIP formulation (without the porogen) and in the polymerized, dried MIP. After the introduction of the particles in the solvent (which defines the initial time of the release experiment), the dispersion was vigorously shaken and homogenized for few seconds and a first sample was extracted with a syringe. The polymer particles were then removed by filtration and the concentration of the "free" caffeine in the liquid phase was determined. Several samples were extracted at regular time intervals.

\section{Batch Rebinding Tests}

As-prepared MIP particles were washed extensively with methanol-acetic acid mixtures (9:1) until caffeine could not be detected in the washing solvent. After each washing step, the particles were isolated from the supernatant using a Hettich centrifuge (Universal 320). Remaining acetic acid was removed by washing twice with pure acetonitrile, and the polymer particles were dried under vacuum at room temperature. Rebinding tests were performed with caffeine solution $(50 \mathrm{mg} / \mathrm{L})$ containing $5 \mathrm{mg}$ of dry MIP powder per $\mathrm{mL}$ of solution. The caffeine concentration in the solution before and after the introduction of the MIP has been used to determine the amount of rebinding sites. The MIP-solvent system was allowed to equilibrate for one day before the measurements of the final concentrations. Further uptake of template by the polymer was not observed with the subsequent increase in the caffeine concentration of the solutions.

\section{RESULTS}

\section{Interactions between the Template and the Functional Monomer}

Caffeine was selected in this work as a model molecular template. Besides being a common additive/ingredient in foods, beverages and pharmaceuticals, caffeine is also a template frequently used in molecular imprinting, ${ }^{21-23}$ and even in MIP release studies. ${ }^{24}$ MIPs for caffeine were prepared according to the reported procedures using methacrylic acid (MAA) as a functional monomer, ethylene glycol dimethacrylate (EGDMA) as the crosslinker and a suitable porogenic solvent (acetonitrile or chloroform). ${ }^{25,26}$ The preparation of a MIP by the non-covalent approach requires the formation of a complex between the functional groups of the monomer and the complementary functional groups of the template prior to the rigidification reactions (cross-linking). In order to confirm the relevance of our model system, an evaluation of caffeinefunctional monomer interactions was performed to ascertain that monomer-template self-assembly does in fact take place in the pre-polymerization mixture. Although caffeine-imprinted polymers have been reported in several studies, little evidence has yet been presented for MAA-caffeine self-assembly during the pre-polymerisation phase.

${ }^{1} \mathrm{H}$ NMR is a powerful technique for studying H-bonding formation between the template molecules and functional monomers. ${ }^{27-30}$ It is used in the present study to gain an insight into the interactions between caffeine and the carboxylic acid
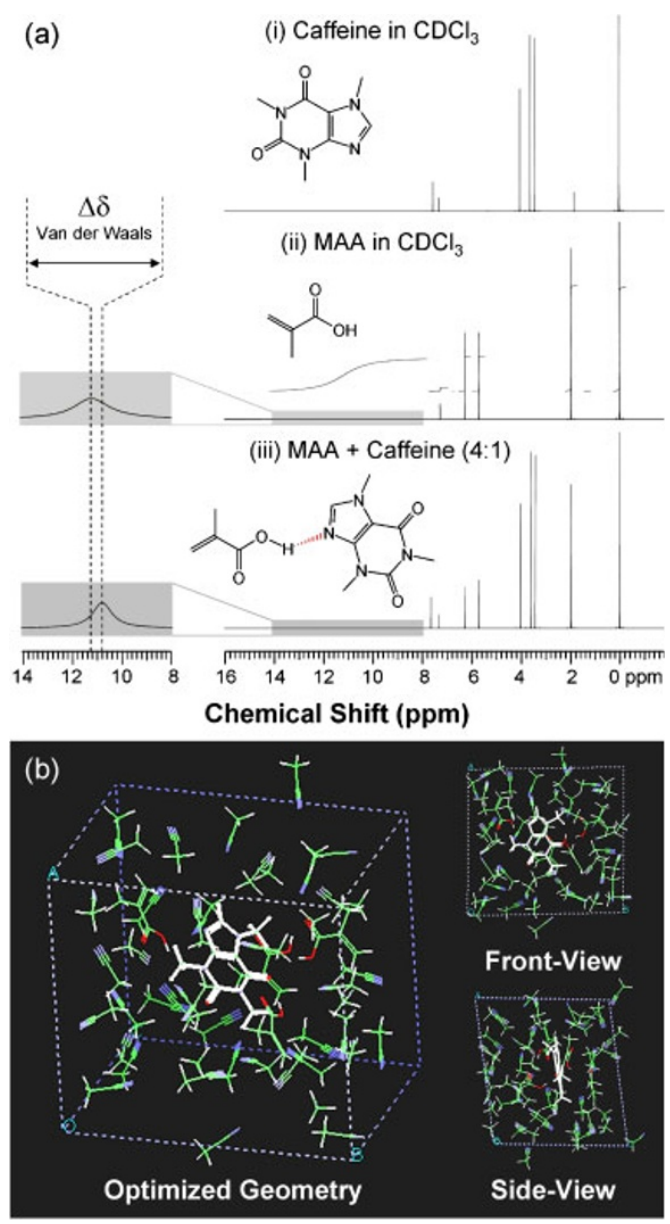

Figure 1. (a) ${ }^{1} \mathrm{H}$ NMR spectra in $\mathrm{CDCl}_{3}$ of pure caffeine $(60 \mathrm{mM})$, pure MAA $(240 \mathrm{mM})$, and a mixture of Caffeine and MAA $(60 \mathrm{mM}, 240 \mathrm{mM})$ (b) Simulated cluster demonstrating the structure of the selfassembled complex between caffeine and MAA in acetonitrile. The simulation has also been performed in chloroform.

group of MAA (Figure 1a). The chemical shift of the MAA proton bound to oxygen is highly dependent on the solvent, temperature, and concentration. ${ }^{31}$ Consequently, the caffeine and MAA solutions have been prepared using a fixed concentration. In the case of pure MAA solution $(240 \mathrm{mM})$, the proton resonance of the $\mathrm{COOH}$ group is located at $11,16 \mathrm{ppm}$. As the concentration of the caffeine in the solution increased to $60 \mathrm{mM}$ (a 1:4 ratio to $\mathrm{COOH}$ groups), this peak moves to $10.88 \mathrm{ppm}$ and then to $10.79 \mathrm{ppm}$ in $120 \mathrm{mM}$ solution (1:2 to $\mathrm{COOH}$ group). Although this peak shift is relatively moderate, it suggests that the proton of the $\mathrm{COOH}$ group is interacting with caffeine (via van der Waals interactions or hydrogen bonding), thus insuring the formation of imprinted cavities with specifically designed size and shape. ${ }^{27-29,31}$

In both porogens, the calculated interactions energy magnitude indicate the following order: Caffeine/MAA $\gg$ MAA/ Porogen $>$ Caffeine/Porogen. The structure of a CaffeineMAA-ACN cluster simulated by molecular dynamics is presented on Figure 1b (calculation conditions: ensemble NPT, pressure $0,5 \mathrm{GPa}$, Temperature $300 \mathrm{~K}$, time $100 \mathrm{ps}$ ). The interaction energy (Molecular Mechanics method, Force field: 
Table II. Physical properties of the MIP particles $(32 \mu \mathrm{m}-50 \mu \mathrm{m})$ in the dry and swollen states ${ }^{\mathrm{a}}$

\begin{tabular}{|c|c|c|c|c|c|}
\hline Items & MIP1 & MIP2 & MIP3 & MIP4 & MIP5 \\
\hline MIP:Porogen volume ratio & $3: 4$ & $3: 2$ & $3: 4$ & $3: 2$ & $3: 1$ \\
\hline Porogen & $\mathrm{ACN}$ & $\mathrm{ACN}$ & $\mathrm{CHCl}_{3}$ & $\mathrm{CHCl}_{3}$ & $\mathrm{CHCl}_{3}$ \\
\hline $\begin{array}{l}\text { Appearance of the monolith in the } \\
\text { polymerisation tube }\end{array}$ & Opaque & Opaque & Translucent & Translucent & Transparent \\
\hline Apparent dry density $\left(\mathrm{g} \cdot \mathrm{mL}^{-1}\right)$ & 0.379 & 0.445 & 0.461 & 0.523 & 0.585 \\
\hline $\begin{array}{l}\text { Apparent volumetric swelling } \\
\text { degree in } \mathrm{ACN}\left(\mathrm{mL} \cdot \mathrm{mL}^{-1}\right)\end{array}$ & 1.180 & 1.103 & 1.499 & 1.247 & 1.189 \\
\hline Specific swelling ${ }^{\mathrm{b}}$ in $\mathrm{ACN}\left(\mathrm{mL} \cdot \mathrm{g}^{-1}\right)$ & 3.110 & 2.479 & 3.252 & 2.382 & 2.033 \\
\hline Specific swelling in $\mathrm{CHCl}_{3}\left(\mathrm{~mL} \cdot \mathrm{g}^{-1}\right)$ & 3.538 & 2.809 & 3.698 & 2.706 & 2.194 \\
\hline Specific swelling in $\mathrm{H}_{2} \mathrm{O}\left(\mathrm{mL} \cdot \mathrm{g}^{-1}\right)$ & 3.245 & 2.524 & 3.295 & 2.419 & 2.077 \\
\hline BET surface area $\left(\mathrm{cm}^{2} \cdot \mathrm{g}^{-1}\right)$ & 273 & 289 & 238 & 219 & 106 \\
\hline Total pore volume $\left(\mathrm{mL} \cdot \mathrm{g}^{-1}\right)$ & 0.698 & 0.526 & 0.372 & 0.316 & 0.162 \\
\hline
\end{tabular}

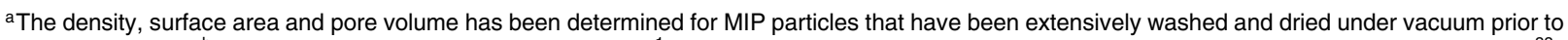
the characterization. ${ }^{b}$ The specific swelling (expressed in $\mathrm{mL} \cdot \mathrm{g}^{-1}$ ) corresponds to the apparent swelling degree divided by the apparent dry density. ${ }^{33}$

Dreiding 2.21) between one molecule of caffeine with 4 MAA molecules in 45 molecules of $\mathrm{ACN}$ is $-58.1 \mathrm{kcal} / \mathrm{mol}$, and clearly indicates that preferential interactions takes place between the template and the functional monomer. Similarly, the interaction energy between one molecule of caffeine with 4 MAA molecules in $30 \mathrm{CHCl}_{3}$ molecules is $-57.3 \mathrm{kcal} / \mathrm{mol}$. These values are typical of van der Waals interactions occurring in the vast majority of non-covalent MIPs, where the monomer-template complexes are rather weak and constantly exchanging in solution. This result can be explained on the basis of the fact that the chemical structure of caffeine does not allow for the establishment of strong hydrogen bonding interactions (as depicted on Figure 1a, Scheme iii). ${ }^{32}$

\section{Preparation of the Molecularly Imprinted Polymers}

Caffeine is not soluble in the monomer cocktail composed of MAA and EGDMA, and the addition of a solvent that dissolves all the components of the pre-polymerization mixture is thus necessary. MIPs formulations with various MIP:porogen volume ratios have been prepared (Table I). Acetonitrile and chloroform were chosen in this work because of their aprotic nature and their low hydrogen bonding ability, two factor that have been shown to be essential in order to maximize the template-monomer interactions. MIP:porogen volume ratios of 3:1, 3:2 and 3:4 were chosen. This range of volume ratio allow for the preparation of polymers with very distinct morphology and release profile, as it will be shown later.

The template:monomer ratio of 1:4 has already been shown to give a good imprinting yield for this system. ${ }^{25,26} \mathrm{~A}$ template:monomer:cross-linker molar ratio of 1:4:20 has been selected for performing the release tests (samples MIP1 to MIP5). Other molar ratios of cross-linker have been envisaged. Polymers with lower concentration of cross-linker (MIP6 in Table I) have a higher loading of template, but were not fully polymerized (as detected by HPLC-UV). Because such samples were susceptible to release the un-reacted monomers in addition of the template, they were not selected for further investigations. Samples with higher concentration of crosslinker (such as MIP7) show a complete conversion, but their loading of template is lower than samples such MIP1.
Polymerization reaction was initiated photo-chemically and conducted for $24 \mathrm{~h}$ at room temperature using a custom-made polymerization device. Polymers prepared in acetonitrile display white opaque appearances. In contrast, monoliths synthesized in chloroform appear translucent in the polymerization tubes. As already observed by Sellergren, ${ }^{33}$ the translucent monoliths progressively turned to opaque after opening of the tubes, probably due to evaporation of the chloroform and the resulting shrinkage of the networks. This shrinkage is reflected in the apparent density of the dry MIP powders (see Table II). Indeed, for a given MIP:porogen volume ratio, polymers prepared in chloroform are always more dense than polymers prepared in acetonitrile, indicating that chloroform evaporation induced a higher shrinkage than acetonitrile evaporation. It should be noted that the gravimetric yield of polymerization of the samples prepared in acetonitrile and chloroform is similar. Therefore the shrinkage may not be attributed to an incomplete polymerization, but rather to a different network structure.

In view of the release tests, it was important to find out if components of the polymer mixture other than the template (for instance MAA or EGDMA) could be released from the unwashed polymers. Gravimetric yields of polymerization were evaluated from the amount of materials extractible from polymerized MIPs. As MAA and EGDMA could not be detected by HPLC-UV, we assume that the polymerization reaction was complete. After polymerization, the bulk polymer was ground with pestle and mortar and dried under vacuum. The particles fraction between 32 and $50 \mathrm{~mm}$ was collected using an analytical sieve shaker and used in the subsequent release test.

\section{Pore Structure Development and Swelling of the Monoliths}

During MIP polymerization, the type and amount of porogen determine the solvation of the growing polymer chains, and are thus crucial parameters in the mechanism of pore structure development. Several studies have been devoted to the pore structure formation in highly cross-linked thermosetting resins, and especially to the influence of the solvating ability of the porogen. ${ }^{33-37}$ The solubility $S$ of the MIP in the 
porogen can be evaluated using formula (1), where $\delta_{\text {MIP }}$ and $\delta_{\text {Porogen }}$ are the solubility parameters of the polymer and the porogen, respectively.

$$
\mathrm{S} \sim\left(\delta_{\mathrm{MIP}}-\delta_{\text {Porogen }}\right)^{2}
$$

A minimization of $\mathrm{S}$ corresponds to a maximization of the solvation of the polymer chains in the porogen. The solubility parameters for the two porogens considered are $\delta_{\mathrm{ACN}}=24.6$ $\mathrm{J}^{1 / 2} \cdot \mathrm{cm}^{-3 / 2}$ and $\delta_{\mathrm{CHCl} 3}=19.0 \mathrm{~J}^{1 / 2} \cdot \mathrm{cm}^{-3 / 2}$. The solubility parameter of the polymer network has been evaluated using the Fedors group contribution theory $\left(\delta_{\mathrm{MIP}}=\left[\sum \Delta \mathrm{e}_{\mathrm{i}} / \sum \Delta \mathrm{v}_{\mathrm{i}}\right]^{1 / 2}\right)$ and were found to be $\delta_{\mathrm{MIP}}=20.18 \mathrm{~J}^{1 / 2} \cdot \mathrm{cm}^{-3 / 2}$. According to eq 1 , it can be concluded that chloroform is a better solvent for the poly(MAA-co-EDMA) network than acetonitrile.

MIP polymerization starts with the formation of nuclei (nano-spheres) that grow around initiator molecules. During polymerization, the growing polymer chains precipitate out of solution either because of extensive cross-linking or because their molecular weights have exceeded the solubility limit of the porogen. In a bad solvent (such as acetonitrile in this study), these nuclei are preferentially swollen by the monomers rather than by the porogen. As a result, these nuclei continue to grow until they agglomerates to form highly cross-linked clusters of nuclei (globules) that further precipitate and form an open and robust 3D network structure, that do not shrink much upon porogen evaporation. On the other hand, in a good solvent such as chloroform, the nuclei are usually smaller and intermolecular cross-linked between nuclei and between globules is favoured. The resulting macroscopic network is more homogeneous and less macroporous. These inter-molecular crosslinks appear to be quite flexible since this type of polymers tends to shrinks upon drying.

The volume of porogen introduced in the pre-polymerisation mixture has also a crucial role in determining the final pore structure. Once the polymer has reach his solubility limit in the porogen, a pure solvent phase is formed (syneresis), and the magnitude of the phase separation is related to the volume of porogen introduced in the initial reactive mixture. Therefore, the final network morphology depends both on the nature and the amount of porogen.

Volumetric swelling degrees of the polymers in acetonitrile, water and chloroform are reported on Table II and illustrated on Figure 2. Several conclusions can be made. At first, polymer swelling degrees decrease following the MIP:porogen volume ratio order 3:4, 3:2 and 3:1. Then, at a given MIP:Porogen volume ratio, polymers prepared in chloroform swell more than polymers prepared in acetonitrile. We have seen before that the polymers prepared in chloroform tend to shrink the most upon drying, and that may explain their higher apparent swelling degree. However, we observe on Table II that a given mass of two MIPs prepared in different porogen but at the same MIP:porogen volume ratio absorb approximately the same volume of solvent (i.e., their specific swelling degree, expressed in $\mathrm{mL}$ of solvent per $\mathrm{g}$ of polymer, is almost equal). This observation reveals that the swelling degree of MIPs strongly correlates with the polymer:solvent volume ratio at

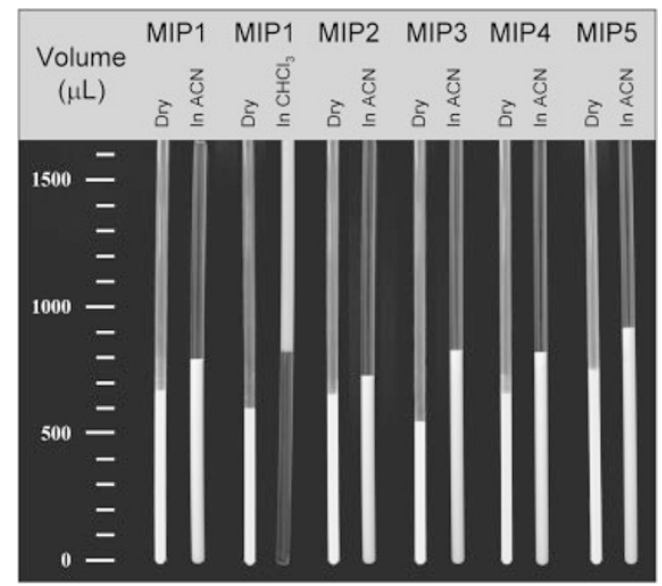

Figure 2. Selected optical micrographs of the volume-calibrated NMR tubes used for measuring the apparent density and the apparent volumetric swelling degree of the MIP particles in ACN, $\mathrm{H}_{2} \mathrm{O}$ and $\mathrm{CHCl}_{3}$. Note the buoyancy phenomena observed in the case of chloroform (i.e., the polymers float on that solvent).

which they have been prepared, independently of the employed porogen. Finally, it can be observed that MIPs swell more in chloroform than in acetonitrile, confirming the fact that chloroform is a better solvent than acetonitrile for the polymer matrix.

\section{MIP Morphology and Porosity in the Dry State}

SEM images of the specimens MIP1, MIP2, MIP3, MIP4 and MIP5 in the dry state are presented on Figure 3a, and clearly highlight the morphological changes induced by the MIP:porogen volume ratio. Samples prepared with a high volume of porogen are more (macro) porous and present morphologies of aggregated beads with small cavities between larger ones (MIP1, MIP3). On the other hand, samples prepared with a smaller amount of porogen display a more compact (gellike) texture and did not present any macropores (MIP5). Polymers prepared in acetonitrile appear more porous than the polymers prepared in chloroform, but that may result from the more pronounced shrinkage of the samples prepared in chloroform. Such a discrepancy may not be observed in the swollen state.

A schematic model of the hierarchical MIP morphology is presented on Figure 3b. The porosity and resulting surface area in MIPs is formed from irregular voids located between crosslinked globules (macropores, 50 to $1000 \mathrm{~nm}$ in diameter), or from the interstitial space of a given cluster of nuclei (mesopores, 2-50 $\mathrm{nm}$ in diameter), or even within the crosslinked nuclei themselves (micropores, $<2 \mathrm{~nm}$ in diameter).

Nitrogen adsorption isotherms and pore size distribution are shown on Figure 4. Desorption isotherms did not always close up with the adsorption branch but form a hysteresis loop (Figure not shown). It is not uncommon that the isotherms of polymers show such an artifact, ${ }^{33}$ because of swelling effects. The total pore volume appears to be directly related to the MIP:porogen volume ratio and to the type of porogen (Table II). At similar MIP:porogen volume ratio, polymers 
(a) Volume Ratio 3:4

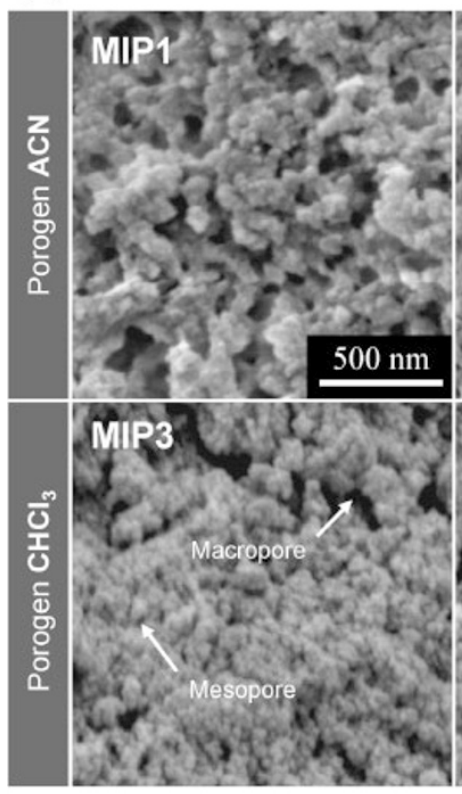

Volume Ratio 3:2

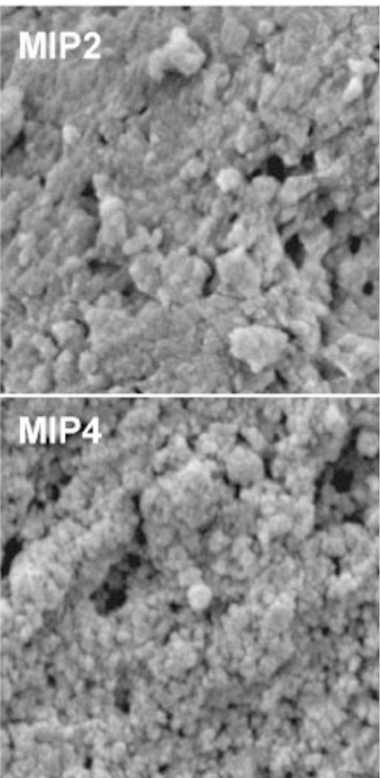

Volume Ratio 3:1

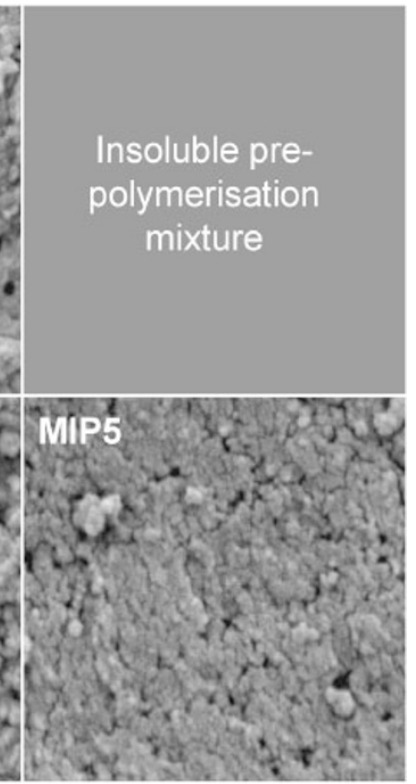

(b)

Hierarchical Morphology of a MIP particle

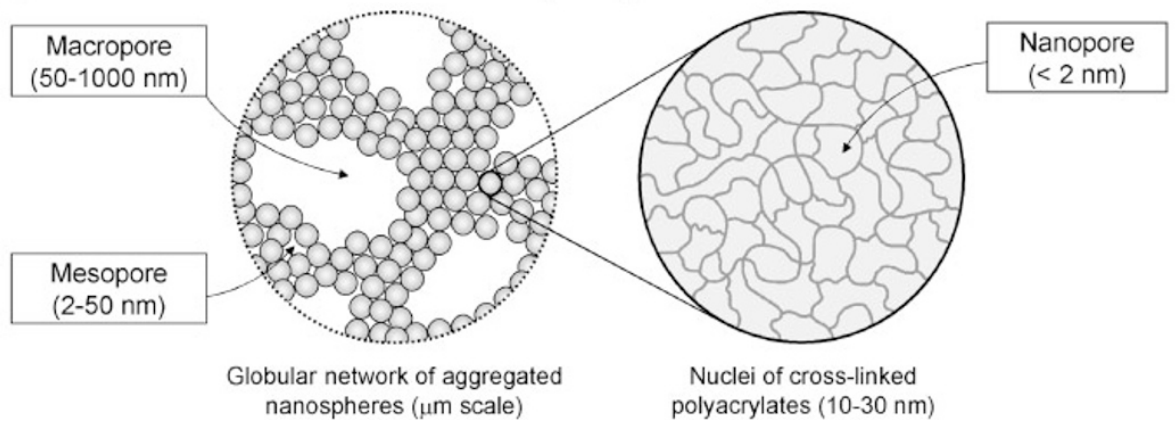

Figure 3. (a) SEM images showing the morphologies of the specimens MIP1, MIP2, MIP3, MIP4, and MIP5, synthesized in different porogenic solvents and with different MIP:porogen volume ratios. (b) Schematic model of the different types of pores observed in the MIP particles: macropore, mesopore and nanopore.

prepared in acetonitrile have a higher pore volume than polymers prepared in chloroform. Once again, this phenomena is a consequence of the more pronounced shrinkage of the samples synthesized in chloroform. The surface area also depends on the MIP:porogen volume ratio. For the samples prepared in chloroform, the surface area of the polymers decreases following the MIP:porogen volume ratios order $3: 4,3: 2$, and $3: 1$. However, for the samples synthesized in acetonitrile, the polymer MIP2 $(3: 2)$ has a higher surface area than MIP1 (3:4). The sample MIP1 display large macropores (see Figure 3a) that could not be detected by nitrogen adsorption measurements. For macroporous sample such as MIP1, another technique (such as $\mathrm{Hg}$ porosimetry) may be required in order to include all the pores.

\section{Procedure of the MIP Release Experiments}

Until now, the release behaviour of MIP have been mainly studied by the "washing and reloading" approach (Figure 5a). Although this approach has the advantage to eliminate most of the non-specifically bonded template, the extensive washing steps and reloading present serious drawbacks for practical applications of MIP containers, such as cost, time and engineering difficulties. Another disadvantage of this technique is the difficulty to optimize the loading capacity of a given MIP. In an attempt to design MIP container that can be used in a more functional way, we have studied the release behaviour of as-prepared MIP particles (Figure 5b).

Release profile of MIPs has been studied as follows. First a dispersion of as-synthesized, dried MIP particles in the release solvent (for instance water) is prepared. The particles were introduced in the solvent, and the dispersion was homogenized for few seconds. A first sample was then extracted with a syringe, defining the initial time of the experiment $\left(t_{0}\right)$. The solid particles were removed by filtration and the concentration of the free caffeine in the solvent was determined with UV-vis spectroscopy by measuring the intensity of the absorbance peak at $272.8 \mathrm{~nm}$ (higher the concentration of caffeine released in the solvent, lower the concentration of caffeine encapsulated in the MIP reservoir). Several samples were extracted at regular time intervals. The dispersion was constantly agitated on a 


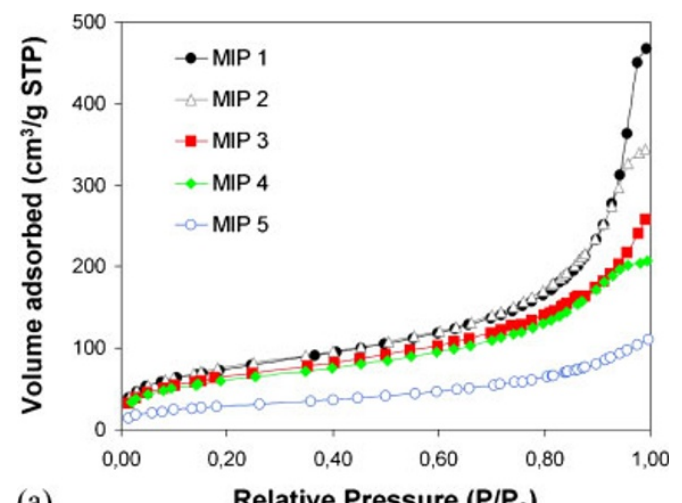

(a) Relative Pressure $\left(P / P_{0}\right)$

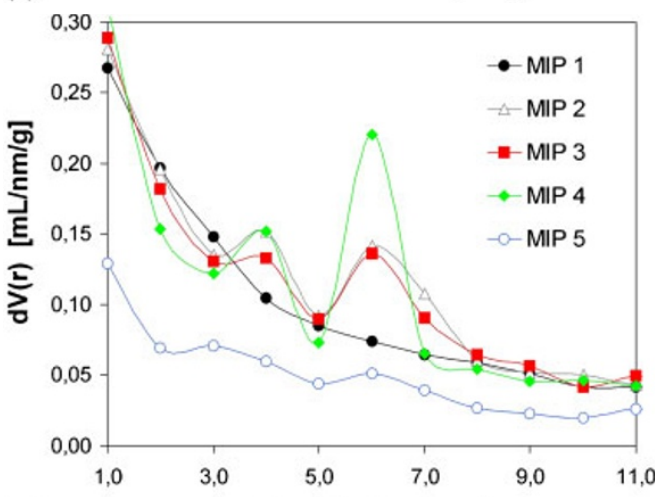

(b)

Pore Radius (nm)

Figure 4. a) Nitrogen adsorption isotherms of polymers MIP1, MIP2, MIP3, MIP4 and MIP5 after helium outgassing at $170^{\circ} \mathrm{C}$. b) Pore size distributions calculated using the BJH model on the adsorption isotherms. $d V(r)$ represents the first derivative of the cumulative pore volume.

rocking Table, and was vigorously shaken before each extraction steps in order to maintain a constant concentration of polymer in the dispersion. The caffeine release fraction $\phi$ is determined at any time by eq 2 , where $\mathrm{Abs}^{\text {Sample }}$ is the absorbance of the sample and Abs ${ }^{\text {Reference }}$ is the theoretical absorbance in case of a complete release.

$$
\phi_{\mathrm{t}}=\mathrm{Abs}^{\text {Sample }} / \mathrm{Abs}^{\text {Reference }}
$$

After a time $t$, the system reach an equilibrium state (constant concentration of template in the solvent) and we define $\Delta \phi^{\text {Release }}$ the fraction of template that has been released between $t_{0}$ and $t$, and $\Delta \phi^{\text {Reservoir }}$ the fraction of template that remained permanently trapped by the macromolecular network. The mass conservation of template in the dispersion during the whole experiment can thus be expressed by eq 3 , where $\phi_{0}$ is the released fraction of template at initial time.

$$
\phi_{0}+\Delta \phi^{\text {Release }}+\Delta \phi^{\text {Reservoir }}=1
$$

The concentration of MIP active sites (in $\mu$ mol per gram of polymer) capable of retaining the template at $t_{0}$, noted $\mathrm{N}^{\text {Retain }}$, is determined from eq 4 where $\mathrm{N}^{\text {Template }}$ is the template concentration in the pre-polymerization mixture (in $\mu$ mol per gram of polymer precursors).

$$
\mathrm{N}^{\text {Retain }}=\mathrm{N}^{\text {Template }} \times\left(1-\phi_{0}\right)
$$

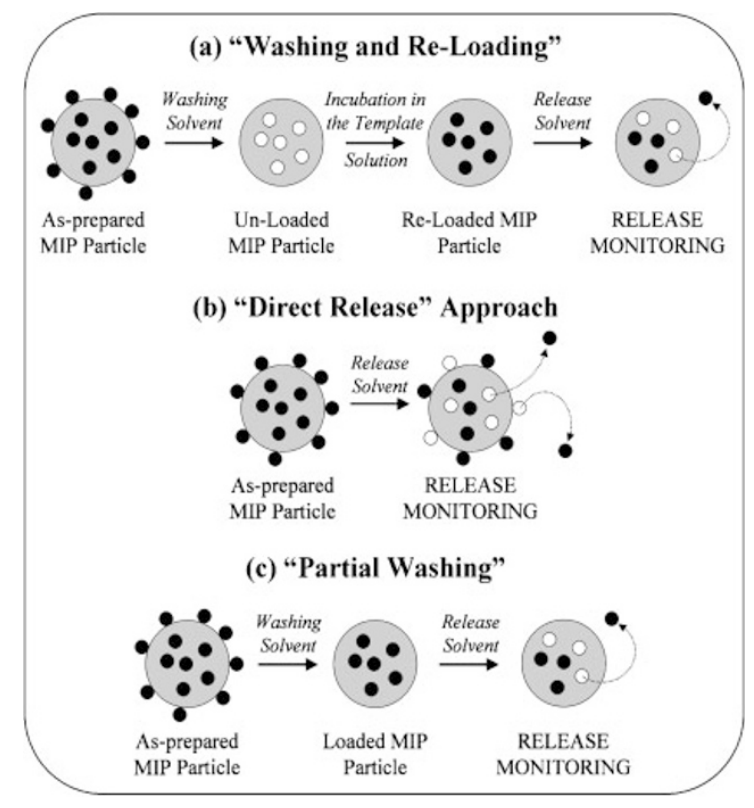

Figure 5. Three different approaches to investigate MIP release: (a) release from washed particles re-loaded with a small amount of template (literature); (b) direct release from as-prepared MIP particles (this work); (c) partial washing approach for the exclusive elimination of the non-imprinted template and optimization of the MIP loading.

Finally, the effective loading capacity of the polymer $\chi$ at $t_{0}$ (in $\mathrm{mg}$ of template per gram of polymer) is determined using eq 5 .

$$
\chi=\mathrm{M}^{\text {Template }} \times \mathrm{N}^{\text {Container }} / 1000
$$

\section{Typical Release Profiles of MIPs}

The release profiles of MIP2 particles in chloroform and in water are displayed on Figure 6b. Our observations indicate that a typical MIP release pattern can be decomposed in three fractions: (large) initial release $\phi_{0}$, sustained release over few hours $\Delta \phi^{\text {Release }}$, and permanently encapsulated fraction $\Delta \phi^{\text {Reservoir }}$. The caffeine release fraction at the initial time may be attributed to the fraction of caffeine that has not been imprinted. Then, a small fraction of template (around 4\%) is slowly released in the solvent over few hours. Finally, a last fraction of caffeine is permanently retained by the polymer and could not be released. This last fraction can be attributed to very good binding sites that effectively hold the template, or to caffeine molecules sterically entrapped into the highly crosslinked polymer networks (non-accessible sites). Release profiles for MIP2 in water and in chloroform follow the same pattern, although $\Delta \phi^{\text {Reservoir }}$ is significantly higher in water than in chloroform. It has already been shown that the nature of the rebinding/releasing solvent significantly affects the performance of MIPs and their binding properties. ${ }^{38}$

Values of $\phi_{0}, \Delta \phi^{\text {Release }}, \Delta \phi^{\text {Reservoir }}$ are reported on Table III for selected dispersions of MIP particles in various solvents. The effect of MIP format (grinded particles or bulk monolith) on the release profiles of MIP2 is presented on Figure $6 \mathrm{~d}$. Interestingly, the initially released fraction $\phi_{0}$ almost vanished 


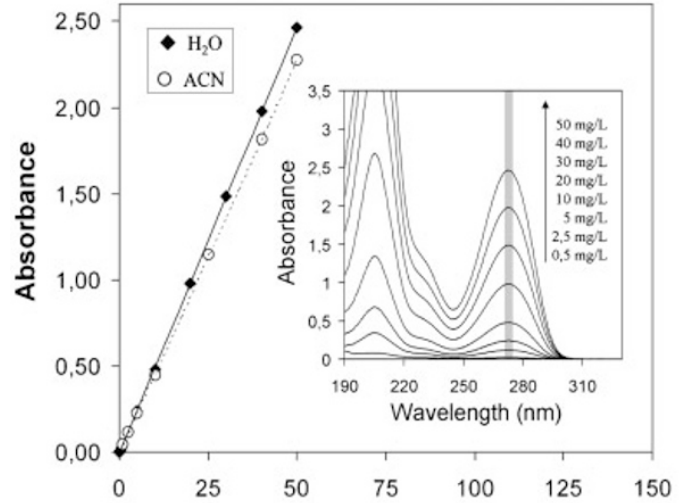

a)

Caffeine Concentration (mg/L)

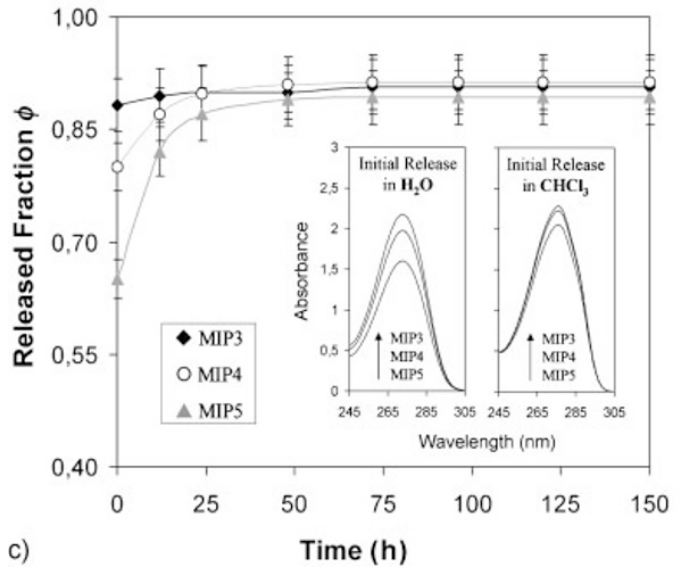

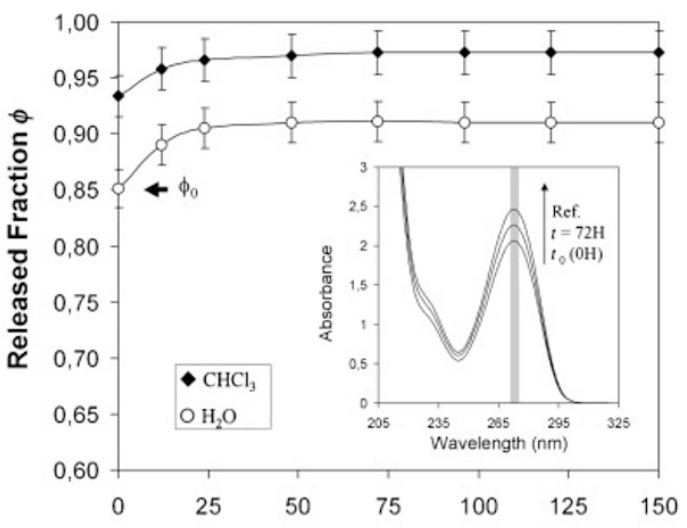

b)

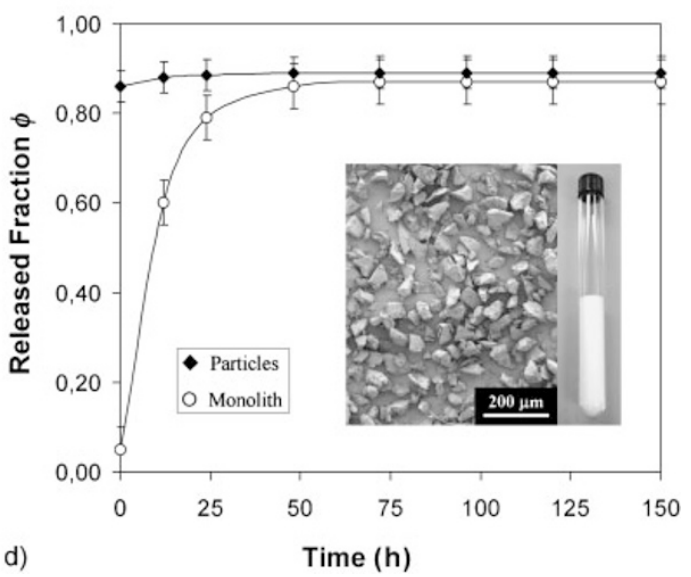

Figure 6. a) Calibration curves for caffeine in water and in acetonitrile. The inset displays UV spectra of standard caffeine solutions in water. b) Release profiles for the sample MIP2 in water and in chloroform. Insert shows the UV spectra of the liquid phases of a dispersion of MIP2 in water at $t_{0}$ and after equilibration of the system $(t=72 \mathrm{~h})$. The reference spectrum of caffeine in water corresponding to a complete release (50 mg. $\left.\mathrm{L}^{-1}\right)$ is also displayed. $\left.\mathrm{C}\right)$ Release profiles of MIP3, MIP4 and MIP5 in water. The inset shows the UV spectra of the liquid phases for dispersions of MIP3, MIP4 and MIP5 in water and in chloroform at the initial time $t_{0}$. d) Influence of MIP format (monolith versus particles) on the release profiles for the sample MIP2 in water. The insert shows a SEM image of sieved particles and an optical image of a polymerized MIP monolith (tube diameter $20 \mathrm{~mm}$ ).

Table III. Results of the release experiments obtained for various dispersions of MIP particles in $\mathrm{H}_{2} \mathrm{O}, \mathrm{ACN}$, and $\mathrm{CHCl}$

\begin{tabular}{|c|c|c|c|c|c|c|}
\hline $\begin{array}{c}\text { Sample } \\
\text { Code }\end{array}$ & $\begin{array}{c}\text { Release } \\
\text { solvent }\end{array}$ & $\phi_{0}(\times 100)$ & $\begin{array}{c}\Delta \boldsymbol{\phi}^{\text {Release }} \\
(\times 100)\end{array}$ & $\begin{array}{c}\Delta \phi^{\text {Reservoir }} \\
(\times 100)\end{array}$ & $\begin{array}{c}\mathrm{N}^{\text {Retain }} \\
\left(\mu \mathrm{mol} \cdot \mathrm{g}^{-1}\right)\end{array}$ & $\chi\left(\mathrm{mg} \cdot \mathrm{g}^{-1}\right)$ \\
\hline MIP1 & $\mathrm{H}_{2} \mathrm{O}$ & 92.52 & 2.61 & 4.86 & 17.05 & 3.31 \\
\hline MIP1 & ACN & 96.95 & 2.53 & 0.52 & 6.94 & 1.35 \\
\hline MIP2 & $\mathrm{H}_{2} \mathrm{O}$ & 86.65 & 2.44 & 10.92 & 30.45 & 5.91 \\
\hline MIP2 & $\mathrm{ACN}$ & 93.86 & 2.06 & 4.08 & 14.00 & 2.72 \\
\hline MIP2 & $\mathrm{CHCl}_{3}$ & 93.37 & 3.93 & 2.70 & 15.11 & 2.94 \\
\hline MIP3 & $\mathrm{H}_{2} \mathrm{O}$ & 88.19 & 2.52 & 9.29 & 26.93 & 5.23 \\
\hline MIP4 & $\mathrm{H}_{2} \mathrm{O}$ & 80.07 & 11.24 & 8.69 & 45.45 & 8.83 \\
\hline MIP5 & $\mathrm{H}_{2} \mathrm{O}$ & 64.98 & 24.31 & 10.71 & 79.88 & 15.51 \\
\hline MIP3 & $\mathrm{CHCl}_{3}$ & 96.37 & 2.57 & 1.06 & 8.28 & 1.61 \\
\hline MIP4 & $\mathrm{CHCl}_{3}$ & 94.01 & 4.98 & 1.01 & 13.67 & 2.65 \\
\hline MIP5 & $\mathrm{CHCl}_{3}$ & 86.75 & 11.19 & 2.07 & 30.23 & 5.87 \\
\hline
\end{tabular}

for the un-grinded monolith. The faster release of the particles as compared to the monolith can be explained by the extraamount of surface area introduced by the grinding process.

\section{Effect of MIP Morphology on the Release Profile}

We have investigated the effect of the MIP porosity on their release profiles. The initially released fraction $\phi_{0}$ is inversely proportional to the MIP:porogen volume ratio, independently on the nature of the porogen or the release solvent used (Figure 7a). This results indicates that the amount of nonimprinted template increases proportionally with the volume of porogen introduced in the pre-polymerization mixture. The released fraction $\Delta \phi^{\text {Release }}$ and encapsulated fraction $\Delta \phi^{\text {Reservoir }}$ both strongly depend on the nature of the release solvent 

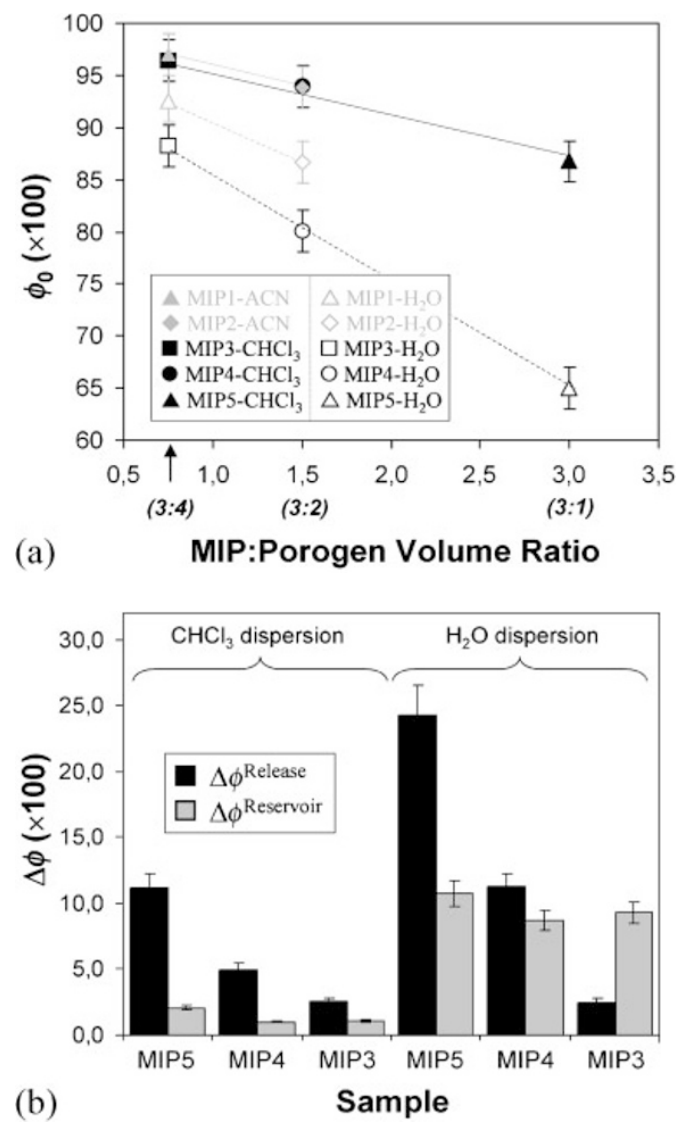

Figure 7. (a) Effect of the MIP:porogen volume ratio on the initial released fraction $\phi_{0}$ for several MIP/solvent dispersions. Grey dots and black dots correspond to polymers synthesized in acetonitrile (MIP1, MIP2) and chloroform (MIP3, MIP4, MIP5), respectively. Straight lines are guides for the eyes. (b) Influence of the MIP:porogen volume ratio on the release fraction $\Delta \phi^{\text {Release }}$ and retained fraction $\Delta \boldsymbol{\phi}^{\text {Reservoir }}$ for dispersions of MIP3, MIP4 and MIP5 in water and chloroform.

(Figure 7b). $\Delta \phi^{\text {Release }}$ significantly increases for denser particles, because the diffusion and mass transfer of caffeine through a denser polymer matrix is hindered. This result suggests that MIP porosity can be used to influence the amount of released template and the release rate. On the other hand, we note that the matrix porosity does not have a great influence on $\Delta \phi^{\text {Reservoir }}$. This observation was surprising, since MIP5 particles were expected to encapsulate more efficiently low molecular weight species, owing to their less macroporous, gellike morphology.

Two MIPs prepared using the same MIP:porogen volume ratio but in different porogens can present significantly different releasing/retaining abilities. The template repartition between $\phi_{0}, \Delta \phi^{\text {Release }}$, and $\Delta \phi^{\text {Reservoir }}$ are reported on Table III for dispersions of MIP2 and MIP4 in water. Those samples have the same compositions and have been synthesised using the same MIP:porogen volume ratio, but with different porogen (ACN for MIP2 and chloroform for MIP4). MIP2 has a greater tendency to retain the template $\left(\Delta \phi^{\text {Release }}<\Delta \phi^{\text {Reservoir }}\right)$ while MIP4 shows the opposite trend $\left(\Delta \phi^{\text {Release }}>\Delta \phi^{\text {Reservoir }}\right)$. This illustrates how tailoring the conditions under which MIPs are prepared can significantly influence their release behaviour.

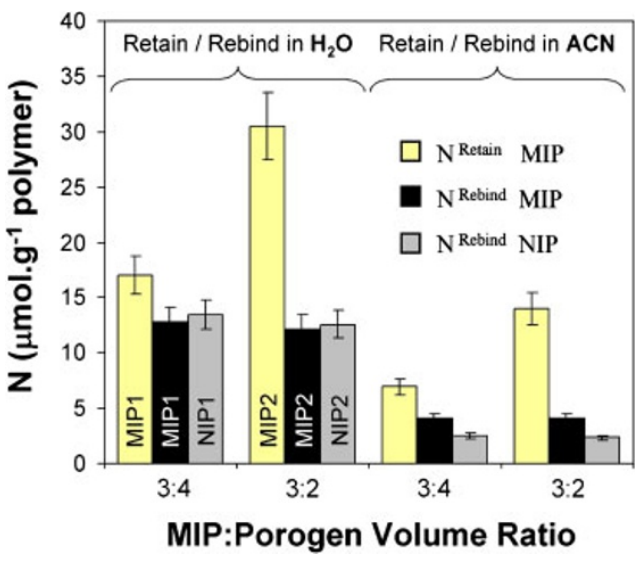

Figure 8. Influence of the MIP:Porogen volume ratio on the number of sites able to rebind caffeine ( $\mathrm{N}^{\text {Rebind }}$ ) for the samples MIP1, NIP1 (MIP:Porogen ratio 3:4) and MIP2, NIP2 (MIP:Porogen ratio 3:2). As a comparison, the numbers of sites that retain the template at the initial time of the release experiments $\left(N^{\text {Retain }}\right)$ are also indicated.

Although the effect of polymer morphology on the retaining behaviour of imprinted organic resins has not been reported before, it should be noted that similar studies have been already performed using inorganic silica matrixes imprinted with various aroma molecules. These studies gave some evidence that the porosity of the inorganic matrix has a strong influnece on both the retaining ability of the silica matrix and the release kinetics of the template. ${ }^{39,40}$

\section{Correlation between Release and Rebinding Experiments}

Sellergren et al. have used an in situ release test for the screening and selection of potential MIP candidates in combinatorial synthesis. ${ }^{41,42}$ In this work, $1 \mathrm{~mL}$ of porogen was added to polymerized mini-MIPs (50 mg each), and the concentration of released template in the supernatant was measured after few hours of equilibration. These authors postulate that the rate of release and the amount of released template correlates with the quality of the binding sites.

Similarly, we attempted to compare our release results with the rebinding properties of the MIPs. The samples NIP1, MIP1, NIP2, and MIP2 (prepared using $\mathrm{ACN}$ as a porogen) were analyzed in conventional heterogeneous batch-rebinding tests in acetonitrile and in water. The numbers of active binding sites able to adsorb the template, noted as $\mathrm{N}^{\text {Rebind }}$ (in $\mu$ mol per gram of polymer), were determined from the batch rebinding studies and are reported on Figure 8. In acetonitrile, the MIP had a higher affinity towards caffeine than the blank polymers (NIP), as a result of the imprinting effect. The specificity of the MIPs vanished in water, presumably due to the competing influence of water molecules. The MIP:Porogen volume ratio (MIP porosity) does not significantly affect the rebinding properties between MIP1 and MIP2.

The numbers of sites that effectively retain the template at the initial time of the release experiment, $\mathrm{N}^{\text {Retain }}$, are also reported on Figure 8. $\mathrm{N}^{\text {Retain }}$ represents the effective template loading of the as-synthesized MIP particles at $t_{0}$, whether 
$\mathrm{N}^{\text {Rebind }}$ corresponds to the maximum loading achievable from physical adsorption. As indicated by Figure 8, the values of $\mathrm{N}^{\text {Retain }}$ are always higher than $\mathrm{N}^{\text {Rebind }}$. Therefore, the molecular complexation between the template and the functional monomers occurring during the MIP synthesis allows the introduction of a higher concentration of template into the MIP than it is possible by simple physical adsorption. ${ }^{18}$ However, considering the rather low concentration of specific rebinding sites in the MIP, we presume that their effect on the global release profile of the system may be rather limited (and absent in the case of release in water). Therefore, MIPs release profiles appear to be controlled mainly by the morphology and format of the caffeine MIP, rather than their molecular recognition properties.

\section{DISCUSSION}

\section{Rebinding Versus Retaining Properties of MIPs}

The major purpose of this study is to conduct a preliminary investigation on the release behavior of as-synthesized MIP particles. Our observations indicate that a typical MIP release pattern can be decomposed in three fractions: (large) initial release, sustained release over few hours, and permanent retention/encapsulation. It is interesting to understand how these different fractions correlate with the structure of the imprinted cavities. Imprinted polymers prepared by the noncovalent route are characterized by binding sites of widely different qualities. ${ }^{43,44}$ A schematic representation of an asprepared MIP particle is illustrated on Figure 9. Within such a complex supramolecular system, the template can be either non-imprinted and dissolved in the porogen phase (9a), weakly adsorbed at the polymer interface (9b), located in un-selective recognition voids (9c), encapsulated in MIP cavities showing good affinity for the template (9d), or permanently entrapped within the mesh of the densely cross-linked network (9e). MIPs synthesized with high MIP/porogen volume ratio display a highly porous morphology favouring a large fraction of template dissolved in the macropores together with a high proportion of template non-specifically adsorbed at the polymer interface. This explain the proportional evolution of $\phi_{0}$ with the MIP:porogen volume ratio observed on Figure 7a.

For the vast majority of non-covalent MIPs, the monomertemplate complexes are rather weak and constantly exchanging in solution. As a result, the population of good re-binding sites for such polymers often corresponds to only $1 \%$ of the amount of template used in the synthesis. ${ }^{49}$ A careful study of the available literature reveals that the role of the good binding sites in MIP release is not clear yet, and still under debate. For instance, Norell and Nicholls prepared non-covalent imprinted MIP particles for the template theophylline (a xanthine with a structure very similar to caffeine) using chloroform as a porogen. ${ }^{45}$ Theophylline-reloaded particles were able to sustain drug release in $\mathrm{pH} 7.0$ phosphate buffer for several hours, especially those loaded with low amounts of theophylline (0.1$2.0 \mathrm{mg} / \mathrm{g}$ ). The increase in release rate observed at greater loadings was attributed to a partial drug adsorption to non-

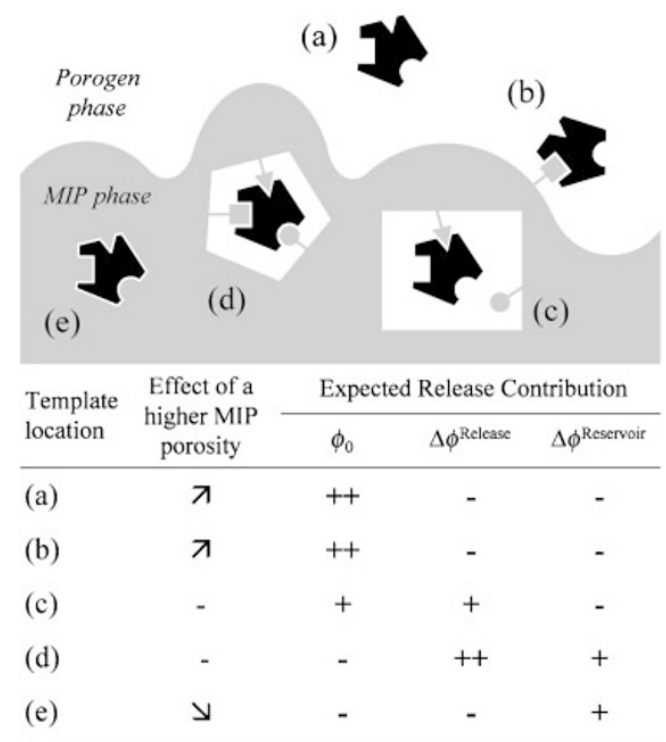

Figure 9. Schematic model of an as-synthesised MIP particle. The template can be either non-imprinted and dissolved in the porogenic solvent (a), weakly adsorbed at the polymer interface (b), located in unselective recognition voids (c), encapsulated in MIP cavities showing good affinity for the template (d) or permanently entrapped within the mesh of the densely cross-linked network (e). The effect of MIP porosity on the population of sites (a-e) is highlighted in the table, together with the expected contribution of the different sites to the releasing/retaining ability of the MIP.

specific binding sites to which it was weakly attached. This hypothesis also explains why the reference (non-imprinted) polymer showed a slightly faster release in some cases. It was observed that the totality of the reloaded template was released after few hours, in other words that $\Delta \phi^{\text {Release }}=1$ and $\Delta \phi^{\text {Reservoir }}=0$. Based on those results, the authors proposed the use of MIP as an oral controlled release drug dosage.

Concurrently, Sreenivasan prepared several MIPs showing high selectivity for various templates such as theophylline ${ }^{46}$ or hydrocortisone. ${ }^{47}$ Those MIPs were prepared using hydroxyethyl methacrylate (HEMA) as a functional monomer and EGDMA as a crosslinker, without the use of a porogenic solvent (non-porous MIPs). The MIPs were successively washed, re-loaded, and subjected to a release test in aqueous methanolic solutions. Interestingly, it was observed that most the template remains permanently encapsulated inside the MIP cavities $\left(\Delta \phi^{\text {Reservoir }} \approx 1\right)$. However, the template can be conveniently recuperated by immersion in a suitable solvent. Those observations suggest that MIPs may be used as a longterm storage medium for analytes.

Because the re-loading approach (Figure 5a) was used in the two studies mentioned above, ${ }^{45,46}$ we expect that in both case the template was mainly adsorbed within good binding sites of the MIPs (9d) and that the effects of loosely bounded template $(9 a-9 c)$ or permanently trapped template $(9 \mathrm{e})$ can be neglected. As results, the discrepancies observed in the studies mentioned above can be solely attributed to the different MIP microenvironments (including the MIP porosity) and to the different binding strength between the polymers and the template theophylinne. 
Such a conclusion is further supported by Piletska et al., who report the development of computationally designed MIPs able to provide the controlled release of the herbicide simazine into water. ${ }^{18}$ Using the direct release approach (Figure $5 b$ ), these authors have shown that the presence of functional monomers is important for polymer affinity (increased polymer capacity) and for controlled release of the template. ${ }^{18}$ Moreover the speed and amplitude of release of the herbicide correlates with the calculated binding characteristics of the MIP (the high-affinity methacrylic acid based polymer released around $2 \%$ of the template whether the low-affinity hydroxylethyl methacrylate based polymer released $27 \%$ of the template over $25 \mathrm{~d}$ ). However, it should be noted that the solubility of simazine in water is very low, and that surely influences both the kinetics and amount of template released. Once simazine has reached its solubility limit, it cannot be released anymore from the MIP, even if the template is adsorbed on the polymer via non-specific interactions. In contrast, in the present study, the template caffeine is quite soluble in all the released solvents employed.

\section{Applicability of MIPs as Reservoirs of Active Substances}

The above results and the survey of the literature demonstrate that MIPs materials may be used either as encapsulation media or as release devices. In view of practical applications of MIP reservoirs, the large initial fraction $\phi_{0}$ released in an uncontrolled manner need to be eliminated. A partial washing approach will be an advantageous solution, eliminating the loosely bonded template and maximizing the concentration of loaded template (Figure 5c). The advantages of MIPs as reservoir in industrial and biomedical applications are closely related to their superior physical and chemical resistances towards various external-degrading factors. MIPs are stable against mechanical stress, elevated temperatures, and high pressures, resistant against treatments with acids, bases, and in a wide range of solvent. ${ }^{48,49}$ In that respect, MIPs containers would have a competitive advantage over current releasing technologies using brittle or soft materials such as hollow microcapsules with liquid cores, ${ }^{50,51}$ or liposomes. ${ }^{52}$ However, in order to use the specificity of the MIP cavities as a driving force for controlling the releasing rate, the affinity and capacity of the best binding sites needs to be significantly improved. The use of stoichiometric or covalent imprinting procedures pioneered by Wulff should contribute to such an increase in reservoir sites. ${ }^{53,54}$ However, this method considerably limits the range of templates available for industrial release applications (very few templates of practical interest allow for such a molecular design, and elaborated synthetic procedures are often required for monomer preparation). Finally, it will be advantageous to develop MIP that can release their template on command, for example following the application of a stimulus such as light ${ }^{24,55}$ or temperature. ${ }^{56}$

\section{CONCLUSION}

With the aid of a model system using caffeine as a molecular template, we have performed preliminary investigations of the influence of polymer morphology on the release behaviour of imprinted resins. It was observed that the initially released fraction is inversely proportional to the MIP:porogen volume ratio. The permanently retained fraction depends on the types of release solvent, but is not much affected by the porosity of the polymer matrix. The above results show that template retention and release in MIPs can be influenced by the polymer porosity, and hence by the MIP:porogen volume ratio of the pre-polymerisation mixture. In other words, higher monomer fraction in the initial preparation process is effective for releasing higher amount of payloads from as-prepared MIPs. Another important aspect of this work is to critically compare our result with the existing literature, and to highlight the current understanding (and knowledge gaps) of MIP release using selected examples.

Overall, MIP release appears to be a very complex phenomenon in which several factors play a role, and a huge amount of work still need to be done before definitive conclusions could be drawn regarding their practical use. Besides porosity, other parameters should be taken into account, such as the binding constant of the template to the polymer, the solubility of the template into the release fluid, the particles shape and size, the temperature, the swelling of the matrix, the molecular weight of the template molecule, or the polymerization conditions. From a conceptual point of view, the contribution of the different types of binding sites on the global releasing process needs to be confirmed. The information generated since three decades about the synthesis of imprinted materials with the optimum performance in separation-based technologies may be a good starting point to create imprinted controlled release systems, although with some subtle differences. For instance, in the case of release applications, the structure of the imprinted cavities should be stable enough to maintain the conformation in the absence of the template, but somehow flexible enough to facilitate the attainment of a fast equilibrium between the release and reuptake of the template in the cavity.

Finally, an important issue for MIP reservoirs development will be the need to find the right market. The market needs to be large enough to justify and generate the investment and commercial pressure that will be required to move imprinted materials from research laboratories to commercial reality. In order to achieve these goals, it is obvious that further collaborations between academic and industrial partners in this field are more important than ever.

Acknowledgment. We gratefully acknowledge Michio Umeda, Tomohiro Kontani, Asami Kubo (Nitto Denko) and Prof. Dirk Vanderzande (Hasselt University) for helpful discussions. We thank the analytical group of Prof. Robert Carleer (Hasselt University) for having performed of the NMR and the SEM experiments. Mr. Hajime Fukunaga (Nitto Denko) is gratefully acknowledged for the development of the photopolymerization device. Finally, the management of Nitto Denko is acknowledged for authorizing the publication of this work. 
Received: April 21, 2009 Accepted: August 10, 2009 Published: October 9, 2009

\section{REFERENCES}

1. F. H. Dickey, Proc. Natl. Acad. Sci. U.S.A., 35, 227 (1949).

2. G. Wulff and A. Sarhan, Angew. Chem., 84, 364 (1972).

3. R. Arshady and K. Mosbach, Macromol. Chem. Phys., 182, 687 (1981).

4. "Molecularly Imprinted Materials Science and Technology," M. Yan and O. Ramström, Ed., Marcel Dekker, New York, 2005.

5. M. J. Whitcombe and E. N. Vulfson, Adv. Mater., 13, 467 (2001).

6. Y. Chen and K. D. Shimizu, Org. Lett., 4, 2937 (2002).

7. P. Manesiotis, A. J. Hall, M. Emgenbroich, M. Quaglia, B. Lorenzi, and B. Sellergren, Chem. Commun., 20, 2278 (2004).

8. R. Mohamed, P. Mottier, L. Treguier, J. Richoz Payot, E. Yilmaz, J. C. Tabet, and P. A. Guy, J. Agric. Food Chem., 56, 3500 (2008).

9. G. Vlatakis, L. I. Andersson, R. Müller, and K. Mosbach, Nature, 361, 645 (1993).

10. G. Wulff, T. Gross, and R. Schönfeld, Angew. Chem., Int. Ed., 36, 1962 (1997).

11. S. C. Maddock, P. Pasetto, and M. Resmini, Chem. Commun., 5, 536 (2004).

12. K. Haupt and K. Mosbach, Chem. Rev., 100, 2495 (2000).

13. D. S. Janiak and P. Kofinas, Anal. Bioanal. Chem., 389, 399 (2007).

14. C. Alvarez Lorenzo and A. Concheiro, J. Chromatogr., B: Anal. Technol. Biomed. Life Sci., 804, 231 (2004).

15. B. Sellergren and C. Allender, Adv. Drug Delivery Rev., 57, 1733 (2005).

16. C. J. Allender, C. Richardson, B. Woodhouse, C. M. Heard, and K. R. Brain, Int. J. Pharm., 195, 39 (2000).

17. H. Hiratani, A. Fujiwara, Y. Tamiya, Y. Mizutani, and C. A. Lorenzo, Biomaterials, 26, 1293 (2005).

18. E. V. Piletska, N. V. Turner, A. P. F. Turner, and S. A. Piletsky, J. Controlled Release, 108, 132 (2005).

19. A. Kydonieus, "Treatise on controlled drug delivery-fundamentals, optimization and applications," Marcel Dekker, New York, 1992.

20. N. Peppas, Z. Hilt, A. Khademhosseini, and R. Langer, Adv. Mater., 18, 1345 (2006).

21. C. I. Lin, A. K. Joseph, C. K. Chang, Y. C. Wang, and Y. D. Lee, Anal. Chim. Acta, 481, 175 (2003).

22. T. Kobayashi, Y. Murakawi, S. R. Puchalapalli, M. Abe, and N. Fujii, Anal. Chim. Acta, 435, 141 (2001).

23. G. Theodoridis and P. Manesiotis, J. Chromatogr., A, 948, 163 (2002).

24. C. Gong, M. H. W. Lam, and H. Yu, Adv. Funct. Mater., 16, 1759 (2006).

25. Y. Jin and K. H. Row, Bull. Korean Chem. Soc., 28, 276 (2007).
26. Y. Jin and K. H. Row, J. Ind. Eng. Chem., 3, 494 (2006).

27. B. Sellergren, M. Lepistö, and K. Mosbach, J. Am. Chem. Soc., 110, 5853 (1988).

28. M. J. Whitcombe, L. Martin, and E. N. Vulfson, Chromatographia, 47, 457 (1998).

29. J. Svenson, J. G. Karlsson, and I. A. Nicholls, J. Chromatogr., A, 1024, 39 (2004).

30. I. Idziak, A. Benrebouh, and F. Deschamps, Anal. Chim. Acta, 435, 137 (2001)

31. Z. Li, M. Day, J. Ding, and K. Faid, Macromolecules, 38, 2620 (2005).

32. C. M. Philip and B. Mathew, J. Macromol. Sci., Part A: Pure Appl. Chem., 45, 335 (2008).

33. B. Sellergren and K. J. Shea, J. Chromatogr., 635, 31 (1993).

34. F. Svec and J. M. J. Fréchet, Anal. Chem., 64, 820 (1992).

35. G. P. Gonzalez, P. F. Hernando, and J. S. Durand Alegria, Anal. Chim. Acta, 557, 179 (2006).

36. A. Guyot, in "Synthesis and separations using functional polymers," D. C. Sherrington and P. Hodge, Ed., John Wiley \& Sons, New York, 1989.

37. F. Svec and J. M. J. Frechet, Science, 273, 205 (1996).

38. W. Dong, M. Yan, Z. Liu, G. Wu, and Y. Li, Sep. Purif. Technol., 53, 183 (2007).

39. S. R. Veith and S. E. Pratsinis, J. Agric. Food Chem., 52, 5964 (2004).

40. S. R. Veith, E. Hughes, and S. E. Pratsinis, J. Controlled Release, 99, 315 (2004).

41. F. Lanza and B. Sellergren, Macromol. Rapid. Commun., 25, 59 (2004).

42. F. Lanza and B. Sellergren, Anal. Chem., 71, 2092 (1999).

43. M. Rampey, R. J. Umpleby, G. T. Rushton, J. C. Iseman, R. N. Shah, and K. D. Shimizu, Anal. Chem., 76, 1123 (2004).

44. A. Katz and M. E. Davis, Macromolecules, 32, 4113 (1999).

45. M. C. Norell, H. S. Andersson, and I. A. Nicholls, J. Mol. Recognit., 11, 98 (1998).

46. Sreenivasan, Bioseparation, 10, 395 (2001).

47. Sreenivasan, Angew. Makromol. Chem., 246, 65 (1997).

48. J. Svenson and I. A. Nicholls, Anal. Chim. Acta, 435, 19 (2001).

49. J. D. Marty and M. Mauzac, Adv. Polym. Sci., 172, 1 (2005).

50. H. N. Yow and A. F. Routh, Soft Matter, 2, 940 (2006).

51. W. Tong, C. Gao, and H. Möwhald, Colloid Polym. Sci., 286, 1103 (2008).

52. O. Zelphati and F. C. Szoka, J. Liposome Res., 7, 31 (1997).

53. G. Wulff and K. Knorr, Bioseparation, 10, 257 (2001).

54. C. Lübke, M. Lübke, M. J. Whitcombe, and E. N. Vulfson, Macromolecules, 33, 5098 (2000).

55. C. Gong, K. L. Wong, and M. H. W. Lam, Chem. Mater., 20, 1353 (2008).

56. V. S. Pande, A. Y. Grosberg, and T. Tanaka, Proc. Natl. Acad. Sci. U.S.A., 91, 12976 (1994). 